\title{
Colaboraciones especiales
}

\section{La mirada del cine al cáncer (I): arte, ciencia y conciencia}

\author{
J. González de Dios ${ }^{a}$, M. Tasso Cereceda ${ }^{b}$, B. Ogando Díaz
}

Publicado en Internet:

17-diciembre-2012

Javier González de Dios: javier.gonzalezdedios@gmail.com
${ }^{a}$ Servicio de Pediatría, Unidad de Neonatología. Hospital General Universitario de Alicante. Departamento de Pediatría. Universidad Miguel Hernández. Alicante. España. Autor del libro Cine y Pediatría: una oportunidad para la docencia y la humanización en nuestra práctica clínica • bservicio de Pediatría, Unidad de Oncología Pediátrica. Hospital General Universitario de Alicante. Alicante. España. Magíster en Bioética y en Medicina Humanitaria • 'Médico de Familia. CS Casa de Campo. Madrid. España. Magíster en Bioética y en Cuidados Paliativos.

El objetivo de este artículo es revisar la presencia de patologías oncológicas en el cine con el fin de identificar los principales trastornos de cáncer que han sido retratados en él. Del mismo modo, se intenta describir la praxis médica y la relación entre médico y paciente, así como las experiencias del paciente y la familia, la adaptación social y la intervención de otros profesionales de la salud alrededor de la enfermedad oncológica.

Se revisan algunas de las películas más significativas sobre el cáncer en adultos. Se analiza una muestra representativa de 41 películas en las que el cáncer tiene distinta representatividad, clasificada como "puntual" (8 películas), "argumental" (22 películas) o "relevante" (11 películas) en la película.

El cine puede ayudar a los profesionales de la salud que trabajan alrededor del cáncer, a los pacientes, a la familia y a la sociedad. En ocasiones, el cáncer que se muestra en las películas es diferente de la realidad: las películas prefieren los pacientes más jóvenes y de mayor clase social, así como los cánceres más fotogénicos. Los síntomas, las pruebas diagnósticas y los tratamientos tienden a reflejar la realidad, sobre todo en las películas argumentales producidas en las últimas décadas. Algunas de estas películas puede ser un recurso de primera mano para la formación de profesionales de la salud.

- Cáncer

- Cine

- Contexto social

- Enfermedades

- Oncología

- Relación médicopaciente

El cine, una oportunidad para hablar con arte, ciencia y conciencia de la enfermedad oncológica y, sobre todo, de los pacientes con cáncer y su entorno. Una oportunidad para mejorar la relación profesional-paciente, para mejorar la humanización y para abrir el debate bioético.

\section{The look of the cinema to cancer (I): art, science and consciousness}

The aim of this article is to review the presence of oncological pathologies in the cinema with a view to identifying the main cancer disorders that have been portrayed in films. Likewise it also intends to describe the medical praxis that is employed, the relationship between physician and patient, how the experiences of the patient and the family are represented, the adaptation to social situations, and the intervention of other health care professionals related with cancer patients.

Some of the most significant films about adulthood cancer are reviewed. A representative sample of 41 films was analyzed in which the cancer had a "prompt" (2 films), a "relevant" (22 films) or a "plot" character (11 films).

Cinema has helped to shape certain ways of thinking about the health care professionals who work with patients, the importance of support from the family and the social role, among other things. The

Key words:

- Cancer

- Cinema

- Social context

- Diseases

- Oncology

- Physician-patient relationship cancer showed by films is, sometimes, different from reality: movies prefer younger patients, higher social class and the most photogenic cancer's locations. The symptoms, diagnostic tests and treatments tend to reflect reality, especially in "plot" movies produced in the last decades. Some of those films may be a first hand resource for training health professionals.

Cinema is an opportunity to talk with art, science and consciousness about oncological diseases; cine$\mathrm{ma}$ is a tool to talk about cancer patients and their environment. Cinema is an opportunity to improve the relationship between health professionals and patients, to improve the humanization and to open the bioethical debate. 


\section{EL CINE, UN LUGAR DE ENCUENTRO PARA LA EDUCACIÓN EN MEDICINA}

En los últimos años, la docencia está siendo sacudida por una imperiosa necesidad de adaptación y evolución. La educación encuentra en la estética artes y humanidades- un aliado imprescindible. Educar desde la estética no es querer anclar en la emoción y en la sensibilidad todo el cuerpo de conceptos necesarios para alcanzar la madurez personal. Lo que se pretende es provocar la reflexión sobre los valores individuales, condición imprescindible para cualquier intento de construcción de la personalidad. En este sentido, las técnicas audiovisuales constituyen uno de los métodos más poderosos de difusión de conocimientos, de desarrollo de aptitudes y de creación de actitudes ${ }^{1}$.

El cine, con su imagen y sonido, es un despertador de emociones dentro de la "cultura del espectácuIo". Al igual que otras artes (literatura y teatro,), poesía ${ }^{4}$, ópera ${ }^{5}$, etc.), el cine se convierte, y por méritos propios, en un recurso útil en el universo de la educación médica ${ }^{6-12}$. Porque el cine es posiblemente la manifestación artística más completa a la hora de plasmar una enfermedad: se ve, se oye y se siente. Gracias al cine, podemos aprender más (de enfermedades y, sobre todo, de enfermos y sus familias) y enseñar mejor, porque con cine se desarrolla una deliberación narrativa ${ }^{13,14}$.

Los profesionales de la salud han comenzado a investigar qué pacientes y enfermedades trata el cine y cómo las trata, pero sobre todo ha servido como foro de aprendizaje. Pues el cine (y también el vídeo y la televisión) es un medio de gran impacto para informar, para divulgar mensajes, para sentir emociones, para abrir debates bioéticos en el entorno de la salud y la enfermedad, para conocer a médicos, familiares y sanitarios, etc. La utilización del cine como recurso educativo en estos tiempos dominados por la imagen (la denominada "pantallocracia") es una posibilidad más que real, quizás no explotada en toda su dimensión en la práctica clínica en aquellos lugares donde se forman los sanitarios, bien en el pregrado (aulas de la facultad) o en el postgrado (hospitales y centros de
Atención Primaria). Si a esto se suma el hecho de que la enfermedad ha sido siempre un referente permanente en el cine y de los cineastas, comprobamos que hay muchas películas que pueden servir de base educativa.

El cine se convierte en un método ideal para mostrar una mirada holística de la salud y la enfermedad. Además, el cine y los cineastas nunca se han ocupado solo de enfermedades, sino siempre de enfermos, de su vida, de su lucha por sobrevivir, de sus circunstancias personales y de su mirada humanitaria e integral, que los sanitarios podemos y debemos utilizar para una labor educativa ${ }^{15}$. La formación humanística y ética del médico es una necesidad indiscutible. Pero los estudiantes y los jóvenes médicos (y los no tan jóvenes) presentan una curiosa convivencia entre abundantes conocimientos técnicos y escasa formación humanística. En la mayoría de las facultades de Medicina, los estudiantes han sido formados en enfermedades, no en enfermos ${ }^{16}$. No parece que el examen MIR y la formación MIR contribuyan a mejorar sustancialmente este aspecto. Aún hoy, no es infrecuente que algunos médicos no contemplen a sus pacientes como una persona (con sentimientos, con familia, con un proyecto de vida particular), sino más bien como un conjunto de órganos y patologías ${ }^{17}$. Por ello, debemos aprovechar que el cine puede contribuir de una forma excelente a la formación cognitiva, emocional-sentimental y moral de las personas mediante la vía de la imaginación. Tres funciones esenciales cabe atribuir al cine desde el punto de vista de la educación ${ }^{14}:$ 1) función experiencial: pues amplía nuestro mundo y otros puntos de vista, ensancha nuestra experiencia; 2) función ilustrativa-pedagógica: pues ilustra un determinado problema o pone de relieve un conflicto, y 3) función constructiva-deliberativa: pues contribuye al desarrollo de la autonomía, la crítica y la reflexión, en busca de una toma de decisiones responsable y prudente.

De ello ya hablamos en dos artículos previos en esta revista ${ }^{18,19}$ y remarcamos la potencial utilidad del cine en la docencia y formación humanística y ética del médico. Porque el cine es una afición que 
puede ser más que un entretenimiento, y que puede transmitir conocimientos, vivencias, experiencias... y despertar sentimientos en el espectador, quien a su vez interpreta la narración desde sus experiencias previas, al tiempo que se siente afectado por ella y la incorpora a su vida como una experiencia más propia ${ }^{20}$

\section{EL CINE, UNIVERSIDAD DE LAS EMOCIONES EN LA SALUD Y EN LA ENFERMEDAD}

No existe un género de cine dedicado a la Medicina. Pero la medicina sí es un tema recurrente en el cine, enfocada desde múltiples puntos de vista. La utilización del cine por parte de los profesionales de la salud es un reconocimiento de las posibilidades que ofrece el séptimo arte como recurso para, al menos, alguno de estos apartados: el aprendizaje de actitudes en la atención a los enfermos, la revisión de diferentes patologías, el análisis de las relaciones entre pacientes y profesionales, la identificación de las diferencias socioculturales en la vivencia de la enfermedad, etc. ${ }^{21}$. Y el cine permite la educación de la afectividad, pues se ha valorado su potencial utilidad como herramienta en la enseñanza de la Medicina, especialmente para conocer la condición humana y mejorar la relación médico-paciente.

Se ha confirmado, por experiencias previas, que el cine es un recurso educativo de alto impacto, si bien requiere cautela y conocimiento para su buen uso. No hace falta solo buena voluntad, sino adecuados conocimientos, habilidades y actitudes en el campo de la docencia audiovisual y una relación de amor al cine. Esta universidad de las emociones en la salud y la enfermedad ya dispone de experiencias consolidadas en distintas universidades: Salamanca ${ }^{1,22}$, Pompeu Fabra ${ }^{17,23,24}$, Santiago de Compostela ${ }^{25}$, Buenos Aires ${ }^{15,20}$, etc.

Es necesario potenciar una educación sentimental, educar los sentimientos, educar la afectividad. El cine provoca emociones y, sobre todo, ofrece la posibilidad de contemplarlas y compartirlas en discusión abierta, abriendo caminos para una verdadera reconstrucción afectiva. La convivencia virtual del cine amplía las oportunidades y experiencias, provoca otras vivencias desde la pantalla. A través de las narraciones (reales o ficticias) se reflejan en las películas sus propios conflictos y, a través de estas vivencias virtuales, se provoca una actitud reflexiva que fácilmente se guarda en el ámbito de la memoria afectiva ${ }^{26,27}$.

Prefiguración, configuración y refiguración son los tres momentos de la experiencia narrativa, también aplicable al cine ${ }^{14}$ : 1) prefiguración es la experiencia previa, es decir, las creencias, valores, actitudes, prejuicios ante una enfermedad o una situación concreta; es el "antes de..." ver una película; 2) configuración es la experiencia imaginada, es decir, el mundo de la ficción que nos proporciona la película, y 3) refiguración es la experiencia interpretada, es decir, la conjunción entre el mundo de ficción y el mundo del espectador; es el "después de..." ver una película.

Casi cualquier patología médica ha tenido su hueco en la gran pantalla. En algunas películas, las enfermedades se utilizan de modo contextual, para dar verosimilitud al argumento; en otras, son el centro del argumento. Según García-Sánchez, coeditor de la revista Medicina y Cine ${ }^{11,22}$, las películas se pueden clasificar en: "saludables" (no hay rastro de enfermedad), "puntuales" (solo aparece alguna alusión a la enfermedad), "relevantes" (el proceso mórbido acompaña de modo constante a uno o varios de los protagonistas) y "argumentales" (la película se centra en el impacto que causa la enfermedad).

De una forma general, podemos decir que algunas patologías médicas son especialmente proclives a ser tratadas por el cine (de forma puntual, relevante o argumental) y, entre ellas, destacan las patologías psiquiátricas, infecciosas, pediátricas y, sin duda, las patologías oncológicas.

\section{EL CÁNCER EN EL CINE: PROTAGONISTA PRINCIPAL O SECUNDARIO}

Según la Organización Mundial de la Salud, "cáncer es un término genérico para un grupo de más de 100 enfermedades que pueden afectar a cual- 
quier parte del organismo". El cáncer sigue siendo, pese a los espectaculares avances en los campos de la prevención, el diagnóstico y el tratamiento de estas entidades, una de las principales causas de mortalidad en el mundo. Aunque hay muchos motivos para la esperanza, el diagnóstico de "cáncer" sigue provocando una de las reacciones psicológicas más duras entre personas afectadas y sus familiares. En España, como en el resto de países, los datos son escalofriantes: más de 130000 nuevos casos de cáncer registrados cada año y 90000 fallecimientos.

El cáncer es un filón para el cine, en películas de muy distinto calado: algunas películas dignas y respetuosas, llenas de valores; otras melodramáticas en busca de la lágrima fácil; algunas obras de arte y otras fácilmente olvidables. Siguiendo el esquema propuesto en el estudio de Icart-Isern et al. ${ }^{21}$, las películas sobre cáncer se clasificaron, según la importancia que tiene el cáncer en el guión, en puntuales, relevantes y argumentales:

- Las películas puntuales fueron aquellas que ofrecían al menos tres secuencias sobre una manifestación clínica, prueba diagnóstica o tratamiento asociados al cáncer, que afectaba a alguno de los personajes, aunque no fuera el protagonista.

- Las películas relevantes fueron aquellas en las que la/el protagonista principal tenía cáncer, del que se describían manifestaciones clínicas, se practicaba alguna prueba diagnóstica y/o se aplicaba algún tratamiento; estas situaciones se producían en al menos cuatro secuencias cinematográficas.

- Las películas argumentales fueron aquellas que relataban la enfermedad a través de un texto narrativo o discurso en el que el/la protagonista presentaba un diagnóstico de cáncer; se describían las fases del proceso (clínica, diagnóstico, tratamiento y resolución) que se transmitía tanto en el guión literario (historia), como en el guión técnico (encuadres, iluminación, movimiento de la cámara, música, efectos sonoros, etc.).
Sin pretender realizar una revisión exhaustiva y, menos aún, una crítica cinematográfica, exponemos algunos ejemplos. Las películas se clasifican en esos tres bloques y se enumeran por orden alfabético. En cada película se expone el título en español, el director, el año y el país de realización, así como un breve reseña de potencial interés, donde indicamos la tipología de proceso neoplásico del "protagonista".

La selección de las películas ha seguido un doble criterio: un balance adecuado entre interés por el tema abordado (en nuestro caso, el cáncer en alguna de sus dimensiones) y una mínima calidad cinematográfica. Los tres autores firmantes del artículo han contribuido en la confección de este listado, precisando un acuerdo mínimo de dos autores para su consideración.

\section{PELÍCULAS PUNTUALES}

\section{Biutiful (Alejandro González Iñárritu, 2010). México}

Uxbal (Javier Bardem) es un hombre complejo y con problemas, que sobrevive en Barcelona en caída libre. A su caos cotidiano se suma el sentir cerca la muerte debido a un avanzado cáncer de próstata. En este contexto, intenta reconciliarse con su padre, salvar a sus hijos, encontrar la paz y salvarse a sí mismo.

\section{Inocencia (Paul Cox, 2000). Australia y Bélgica}

Andreas (Charles "Bud" Tingwell), un organista y profesor de música jubilado, descubre que su primer amor, Claire (Julia Blake), vive en su misma ciudad. Cincuenta años después de compartir una apasionada historia de amor en la Bélgica de la posguerra, Andreas decide escribirle una carta y así retoman una intensa relación que los Ilevará a disfrutar, ahora en su vejez y casi a las puertas de la muerte (Andreas sufre un cáncer terminal no especificado y se niega a recibir tratamiento), de una nueva época de renacimiento y de emoción. 


\section{La caja china (Wayne Wang, 1997). Estados Unidos}

John (Jeremy Irons), periodista británico afincado en Hong Kong, recibe la noticia de que tiene una leucemia y de que solo le quedan unos meses de vida, más o menos el mismo tiempo que le resta a la colonia británica para ser independiente. En este tiempo, deberá enfrentarse a su vida y a su pasión por Vivian (Gong Li).

\section{La casa de mi vida (Irwin Winkler, 2002). Estados Unidos}

George (Kevin Kline) recibe terribles noticias sobre su salud (un cáncer pancreático) casi al mismo tiempo que es despedido de su trabajo. Por su parte, su ex esposa Robin (Kristin Scott Thomas) sufre la rebeldía del hijo adolescente que dice odiar a su familia y que, poco a poco, se está sumergiendo en el mundo de la droga. Entonces, George decide arreglar su relación con la familia en los pocos meses que le quedan de vida, para lo cual derriban juntos su vieja casa y construyen una nueva.

\section{Magnolia (Paul Thomas Anderson, 1999). Estados Unidos}

Narra unas pocas horas de la vida de un elenco de personajes en nueve tramas paralelas que tienen lugar en Los Ángeles. Historias aparentemente independientes, pero que guardan entre sí una extraña relación: entre otros, un padre moribundo, un policía enamorado, un niño prodigio, un presentador de televisión, una hija adicta a la cocaína, un original gurú televisivo, un enfermo terminal de cáncer de pulmón (qué gran homenaje al actor Jason Robards, que moriría precisamente de cáncer de pulmón como su personaje en la película).

\section{Te estoy perdiendo (Bruce Wagner, 1999).}

\section{Estados Unidos}

Perry (Frank Langella) es un productor de televisión que, recién cumplidos los 60 años, recibe una fatal noticia: tiene cáncer de pulmón y solo le queda un año de vida. Esta circunstancia sacudirá los pilares sobre los que se asienta toda su familia: muchos secretos y mentiras del pasado empezarán a desvelarse en una crisis familiar de consecuencias imprevisibles.

\section{Venus (Roger Michell, 2006). Reino Unido}

Maurice (Peter O'Toole) es un anciano actor que se reúne con otros dos amigos jubilados todos los días en su bar preferido para tomar una copa, hablar de sus recuerdos, compadecerse de la situación mundial e intentar dar algo de sentido a su vida antes de morir. La edad ha pasado factura en sus ya débiles cuerpos y Maurice padece un cáncer de próstata.

\section{PELÍCULAS RELEVANTES}

\section{0/50 (Jonathan Levine, 2011). Estados Unidos}

Adam (Joseph Gordon-Levitt) es un joven de 27 años que es diagnosticado de un extraño tipo de cáncer óseo. Algo desorientado, le comenta lo sucedido del diagnóstico a su mejor amigo, Kyle (Seth Rogen), a su novia Rachael (Bryce Dallas Howard) y a su madre Diane (Anjelica Huston), y cada uno de ellos reaccionará ante la noticia de una forma diferente, y todos, de alguna manera, le ayudarán a afrontar la situación por la que está pasando.

\section{Ahora o nunca (Rob Reiner, 2007). Estados Unidos Dos ancianos con cáncer terminal, Carter (Morgan Freeman) y Edward (Jack Nicholson), de caracteres y mundos distintos, se conocen en el hospital y se hacen amigos. Y deciden iniciar un viaje por carre- tera con una lista de cosas que hacer antes de mo- rir, esas cosas que siempre han deseado, pero el transcurrir de la vida no les permitió hacer.}

\section{Amarga victoria (Edmund Goulding, 1939). Estados Unidos}

Judith (Bette Davis) es una mujer de éxito y una rica heredera de Long Island, que desarrolla un tumor cerebral. Aunque la operación resulta un éxito inicialmente, el tumor resulta ser maligno y ella se va quedando ciega poco a poco. Por ello, Judith in- 
tentará beberse la vida en grandes tragos y va transitando por distintos estados anímicos. Fue esta una de las películas preferidas de la gran Bette Davis.

\section{Cosas que importan (Carl Franklin, 1998).}

\section{Estados Unidos}

La vida de Ellen (Renee Zellweger), una joven periodista que trabaja en un importante periódico de Nueva York, sufre un gran cambio cuando, presionada por su padre (William Hurt), tiene que regresar al hogar familiar para ocuparse de su madre (Meryl Streep), gravemente enferma de cáncer, a la que la quedan tan solo unos pocos meses de vida.

\section{Elegir un amor (Joel Schumacher, 1991). Estados Unidos}

Hilary (Julia Roberts) comienza a trabajar al servicio de Victor (Campbell Scott), un joven de buena familia que padece una leucemia que todos creen incurable. Acaban enamorándose y ella le ayuda a luchar: porque ahora para ellos amar supone luchar por la vida, y disfrutar del amor será luchar juntos contra la enfermedad.

\section{El juego de la verdad (Álvaro Fernández Armero, 2004). España}

A Ernesto (Tristán Ulloa) le diagnostican un tumor cerebral incurable apenas cumplidos 30 años. Sus amigos quieren hacer sus últimos deseos realidad y él pide acostarse con la novia de su mejor amigo, explorando de esa forma las consecuencias de las sinceridad absoluta en las parejas..., sobre todo cuando se confirma que hubo un error diagnóstico.

\section{Enemigos intimos (Fernando Sariñana, 2007). México}

Película que presenta a personajes que enfrentan diferentes crisis: una mujer que no acepta su envejecimiento; una enfermera muda y su madre diabética; una adolescente en coma por un cáncer; y un arquitecto (Demián Bichir) que vive solo para el negocio inmobiliario y a quien un cáncer de riñón transforma su proyecto de vida. Las historias de todos se enlazan a partir de esta última.

\section{Hazme reír (Judd Apatow, 2009). Estados Unidos}

George (Adam Sandler) es un famoso monologuista al que comunican que tiene una leucemia y que le queda poco de vida. A partir de ese momento se replantea su existencia, y va en busca de una segunda oportunidad, de aquello que aporte significado a su vida. Una película que juega con el contraste de la comedia (por la profesión del protagonista) y la tragedia que supone vivir una noticia de ese calado.

\section{La fuente de la vida (Darren Aronofsky, 2006). Estados Unidos}

Tommy (Hugh Jackman) es un oncólogo que intenta hacer retroceder los tumores cerebrales a través de la investigación animal con monos. Su trabajo está motivado por su esposa Izzi (Rachel Weisz), enferma de cáncer, y para ello realiza dos viajes: uno al pasado, a la España del siglo XVI, y otro al futuro, al siglo XXVI; el fin es encontrar el legendario árbol de la vida cuya savia proporciona la inmortalidad.

\section{La fuerza del cariño (James L. Brooks, 1983). Estados Unidos}

Auroa (Shirley MacLaine) y Emma (Debra Winger) son una madre y una hija muy unidas, pero con puntos de vista muy distintos respecto al amor y respecto a la vida. Las ansias de libertad de Emma, casada con un infiel marido y con dos hijos, se ven frenadas por el cáncer de mama que le diagnostican.

\section{La habitación de Marvin (Jerry Zaks, 1996). Estados Unidos}

Bessie (Diane Keaton) y Lee (Meryl Streep) son hermanas, pero siguieron caminos muy distintos en la vida: Bessie, la mayor, se quedó a cuidar de su padre, postrado durante años por un accidente cerebrovascular; y Lee se mudó a otra ciudad con su esposo 20 años atrás y nunca volvió a estar en con- 
tacto con su familia. Todo cambia cuando a Bessie le informan de que padece leucemia (y necesita un trasplante de médula ósea) y llama a su hermana en busca de ayuda.

\section{La noche de las chicas (Nick Hurran, 1998). Estados Unidos}

Jackie (Julie Walters) y Dawn (Brenda Blethyn) son cuñadas y amigas, trabajan y se divierten juntas. Tras ganar un bingo millonario deciden cumplir el sueño de sus vidas: una noche en Las Vegas. Sin embargo, este feliz acontecimiento llega a la vez que conocen que una de ellas tiene un cáncer de mama con metástasis. Dramáticas y cálidas interpretaciones de sus protagonistas, quienes destilan humanismo en un sólido y complejo retrato de la mujer en una película con ciertas dosis "a lo Thelma y Louise".

\section{La puta y la ballena (Luis Puenzo, 2004).}

\section{Argentina y España}

Vera (Aitana Sánchez-Gijón) es una escritora que está perdida en España tras detectarle un cáncer de mama y tener una crisis matrimonial. Varada en la vida, acepta un trabajo en Buenos Aires, donde le realizan una mastectomía y es allí donde encuentra un tema para su próxima novela: una historia de amor entre una prostituta y un fotógrafo.

\section{La suerte de Emma (Sven Taddicken, 2006).}

\section{Alemania}

Max (Jürgen Vogel) es un mediocre empleado de un concesionario de coches, que recibe la noticia de que padece un cáncer terminal de páncreas. La noticia hace que robe a su jefe, pues quiere pasar los últimos días de su vida en algún lugar paradisíaco de El Caribe. Pero accidentalmente conoce a Emma (Jürgen Vogel), una muchacha solitaria que regenta una granja de cerdos. Entre ambos se establece una relación peculiar, con ese contraste sociocultural entre el mundo urbano y el rural, mientras la enfermedad avanza.

\section{Love Story (Arthur Hiller, 1970). Estados Unidos}

Una de las historias de amor por antonomasia en la historia del séptimo arte, con esa frase lapidaria de "amor significa nunca tener que decir lo siento". Oliver (Ryan O'Neal) y Jennifer (Ali MacGraw) se casan en contra del consentimiento familiar. Al estudiarse por no poder tener un hijo, conocen que ella tiene una leucemia aguda de mal pronóstico, lo que desencadena una historia lacrimógena y precede un final trágico.

\section{Mi vida (Bruce Joel Rubin, 1993). Estados Unidos}

Bob Jones (Michael Keaton) es un ejecutivo de éxito al que la vida va a poner a prueba. Casado con Gail (Nicole Kidman), la felicidad le llega con la noticia del próximo nacimiento de su primer hijo. Pero la alegría se limitará cuando, tras unas pruebas médicas, le comunican que padece un cáncer de pulmón y que quizá no podrá llegar a conocer a su hijo.

\section{Noviembre dulce (Pat O'Connor, 2001). Estados Unidos}

Sara (Charlize Theron) inicia una relación cada mes con un hombre diferente, volcándose en él completamente para, al final, separarse irremediablemente al finalizar el plazo, pues ella no quiere implicarse emocionalmente. Durante el mes de noviembre se nos narra su historia de amor con Nelson (Keanu Reeves), una historia que también finaliza. Y todo esto porque Sara guarda un secreto: tiene un linfoma que la abocará a la muerte.

\section{Otoño en Nueva York (Joan Chen, 2000). Estados Unidos}

Narra la historia de amor de la joven Charlotte (Winona Ryder) y del apuesto galán Will (Richard Gere), dos personas muy diferentes por edad, clase social y personalidad. Sin embargo, ella le cuenta un secreto que cambiará en Will su percepción de la vida y del amor: Charlotte tiene un cáncer terminal (neuroblastoma) y su deseo es vivir una gran aventura de amor antes de enfrentarse a su final. 
Quédate a mi lado (Chris Columbus, 1998). Estados Unidos

Triángulo amoroso entre Luke (Ed Harris), su ex mujer Jackie (Susan Sarandon) y su actual novia Isabel (Julia Roberts). La relación de tirantez entre ambas mujeres cambia cuando se conoce que Jackie tiene un cáncer de mama e Isabel va, poco a poco, adquiriendo la responsabilidad de cuidar a los hijos de Jackie.

\section{The Guitar (Amy Redford, 2008). Estados Unidos}

Melody (Saffron Burrows) recibe en el mismo día tres noticias: que su novio la abandona, que la han despedido del trabajo y que está enferma de un cáncer terminal. Ante esta situación, decide perseguir sus sueños durante los dos meses de vida que le restan, retomar lo que dejó perdido en la monotonía para revivir lo que dejo atrás. Y solo una guitarra con la que sueña desde que era niña la acompaña hasta el final.

\section{Tierras de penumbra (Richard Attenborough, 1993). Reino Unido}

Se centra en la relación real que acaeció entre el escritor C. S. Lewis (Anthony Hopkins) y Joy Gresham (Debra Winger), poetisa estadounidense divorciada y gran admiradora suya, y donde se da gran valor a la muerte de esta por un cáncer óseo. Maravillosa historia de amor, basada en la biografía del poeta, cuyo excelente ensayo en carne viva "Una pena en observación" dio origen a la película.

\section{Vivir (Akira Kurosawa, 1952). Japón}

Kanji (Takashi Shimura) es un viejo funcionario público que arrastra una vida monótona y gris; sin embargo, no es consciente del vacío de su existencia hasta que le diagnostican un cáncer gástrico incurable. Con la certeza de que el fin de sus días se acerca, surge en él la necesidad de buscarle un sentido a la vida. Y cuando lo consigue se produce un cambio radical en su actitud respecto a los demás.

\section{PELÍCULAS ARGUMENTALES}

Amar la vida (Mike Nichols, 2001).

Estados Unidos (Fig. 1)

Vivian (Emma Thompson) es una profesora de literatura, soltera y racional, a la que un día diagnostican de un cáncer de ovario metastásico en estadio IV. La película está repleta de monólogos, propios de la adaptación de la obra teatral de la que procede, de extraordinario valor. Monólogos para la reflexión, en los que no faltan los comentarios a los profesionales sanitarios y en los que la Enfermería queda algo mejor parada que los médicos. La acompañamos en el proceso diagnóstico (incluyendo las diversas, variadas y sucesivas pruebas complementarias) y en el tratamiento (en sus distintas facetas y con su diferente agresividad, incluyendo algún tratamiento experimental) y a esa profunda soledad con la que lo vive, debido al fuerte carácter que tiene, que hace que casi no tenga amigos. Escenas para recordar (y que todo sanitario debería ver, al menos para sentir lo que no se debe hacer) son el momento en el que se le comunica el diagnóstico del cáncer o los pases de visitas médicos.

Figura 1. Amar la vida (Mike Nichols, 2001)

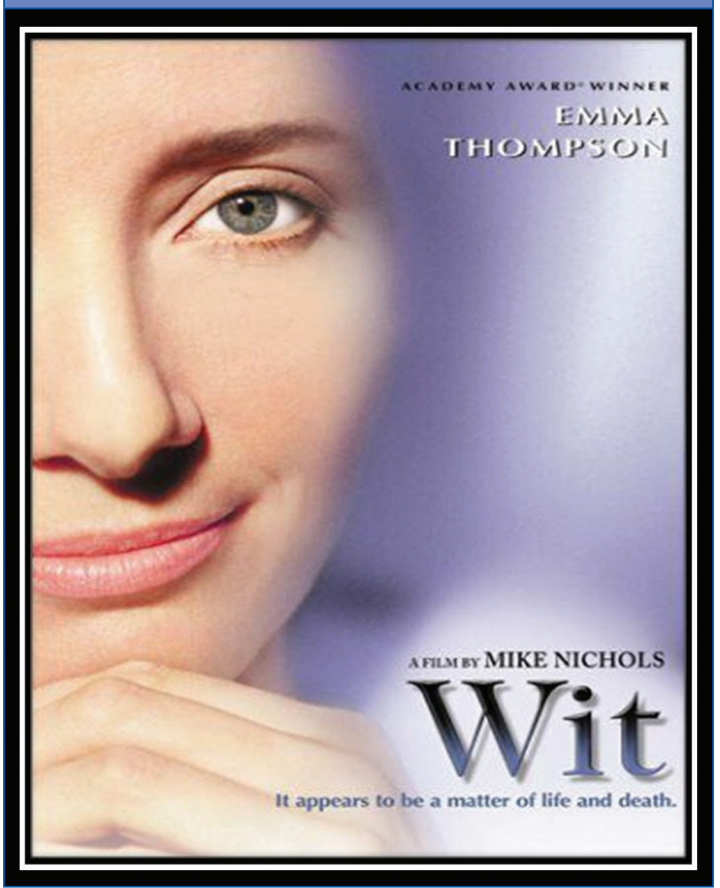


La fuerza de la película (y del texto del que procede) se encuentra en los brillantes diálogos entre la paciente y el personal del hospital: el médico joven y ambicioso, el médico veterano sin sentimientos, la enfermera con algo de humanidad y, especialmente, los monólogos de Vivian mirando a la cámara, dotados de enorme sentido, profundidad y lirismo: "Tengo cáncer, un cáncer insidioso con graves efectos secundarios, perdón, con nocivos efectos secundarios. Tengo un cáncer ovárico metastásico en fase cuatro. La verdad es que no hay fase cinco. Ah, y tengo que ser muy fuerte. Como se suele decir normalmente, es una cuestión de vida o muerte".

\section{Caro Diario (Nanni Moretti, 1993). Italia (Fig. 2)}

Película dividida en tres episodios ("En mi Vespa", "Islas" y "Médicos"), es en la tercera parte en la que el director rueda su experiencia con los vaivenes para llegar al diagnóstico de la causa de un prurito insoportable que, finalmente, resultó ser un tumor del sistema linfático alojado en el pulmón. Nanni Moretti (director y actor) nos ofrece toda su fina ironía para criticar el fracaso del sistema sanitario.

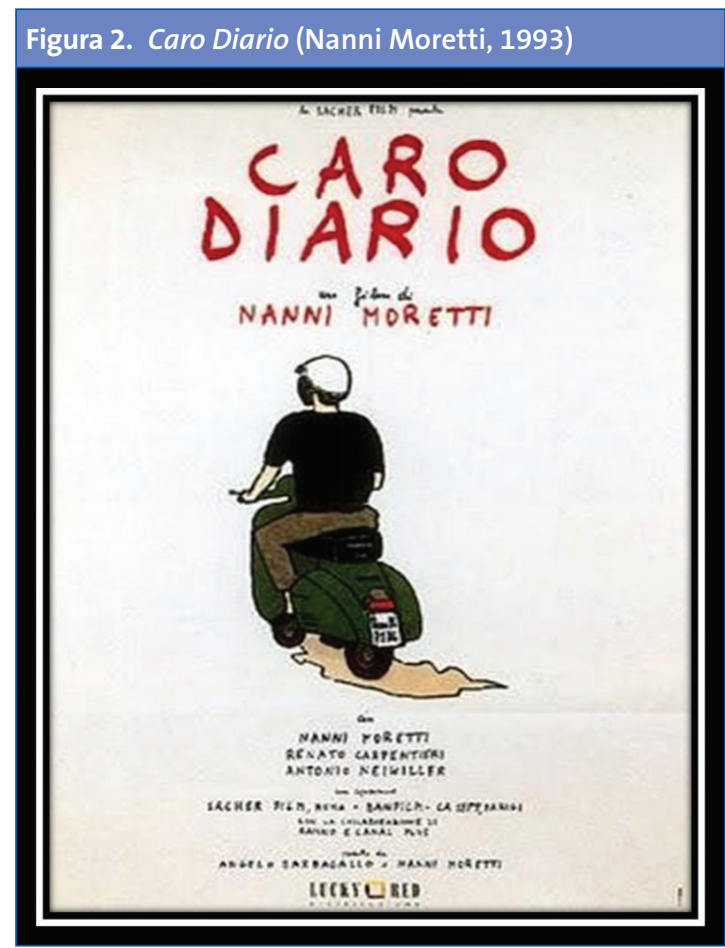

Crazy Sexy Cancer (Kris Carr, 2007).

\section{Estados Unidos (Fig. 3)}

Es la propia actriz, fotógrafa y directora (Kris Carr) la que comparte en esta película documental su experiencia y evolución cuando fue diagnosticada de un hemangioendotelioma epiteliode, un cáncer vascular muy infrecuente que se desarrolló en el hígado y los pulmones. Todo un diario y un viaje en busca del mejor oncólogo y el mejor tratamiento, entre la medicina alopática y las medicinas alternativas, entre la duda y la esperanza, con una dosis de optimismo que acaba transformándose en algo más que una película de una mujer con cáncer.

\section{El Doctor (Randa Haines, 1991).}

\section{Estados Unidos (Fig. 4)}

Jack MacKee (William Hurt), brillante cirujano, rico y con éxito, está convencido de la necesidad de distanciarse emocionalmente de los pacientes: "ES peligroso encariñarse demasiado con los pacientes"; "no conviene volcarse demasiado"; "la misión del cirujano es cortar: entras, lo arreglas y te largas". Su vida cambia cuando un día unas molestias en la

Figura 3. Crazy Sexy Cancer (Kris Carr, 2007)

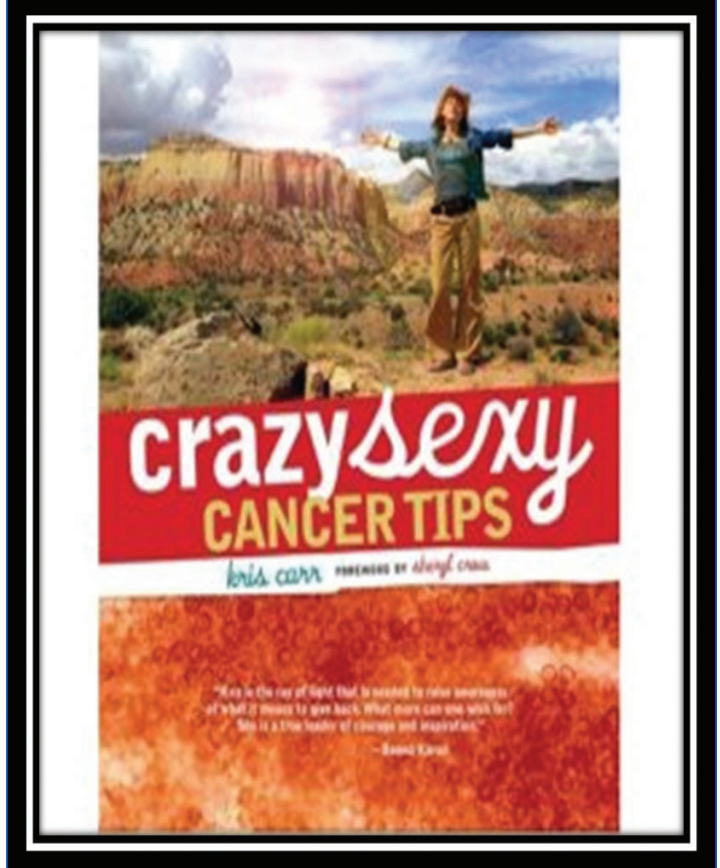




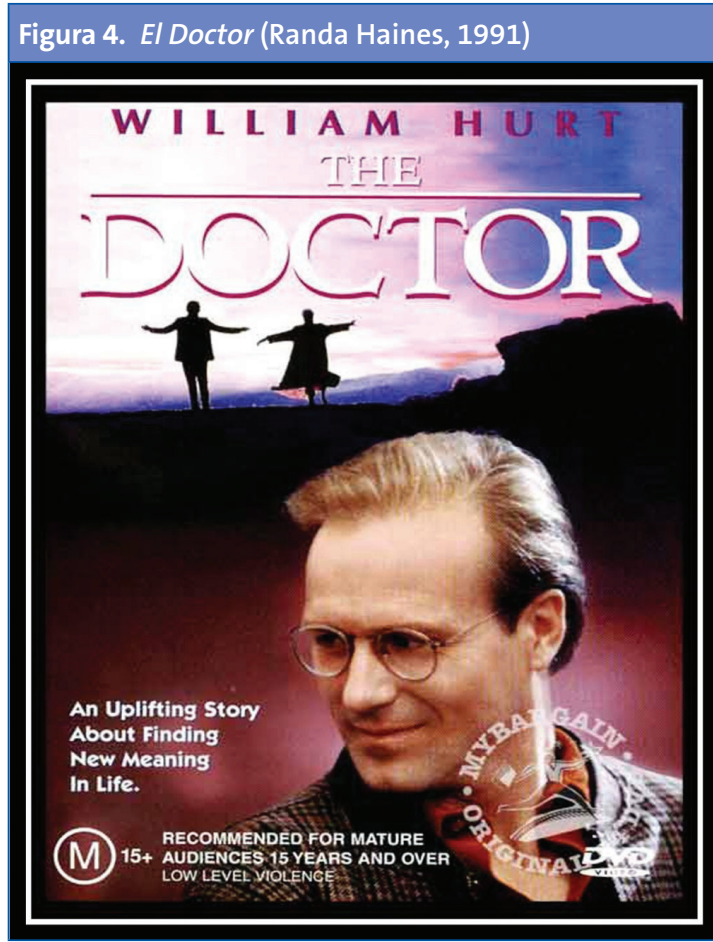

garganta le llevan a la consulta de una compañera, quien le diagnostica un cáncer de laringe. Jack será quien padezca ahora, como cualquiera de sus enfermos, las desagradables consecuencias de la burocracia y la frialdad de los médicos que él tanto defendía. Escenas especialmente reveladoras al final de la película, cuando tras las experiencias vividas durante su enfermedad (y como enfermo), recibe a sus nuevos residentes y les obliga a convertirse en pacientes durante un día completo: visten la misma indumentaria que los pacientes, siguen la misma dieta y son sometidos a algunos de sus procedimientos diagnósticos. El último mensaje del Dr MacKee es reconocer que los pacientes se sienten asustados, avergonzados y vulnerables y confían tanto que, en realidad, ponen su vida en las manos de sus médicos.

\section{El tiempo que queda (François Ozon, 2005).}

\section{Francia (Fig. 5)}

A Romain (Melvil Poupaud), un joven fotógrafo de moda, homosexual, egocéntrico y arrogante, le diagnostican un cáncer de hígado con metástasis y sin esperanzas de curación. Su primera reacción

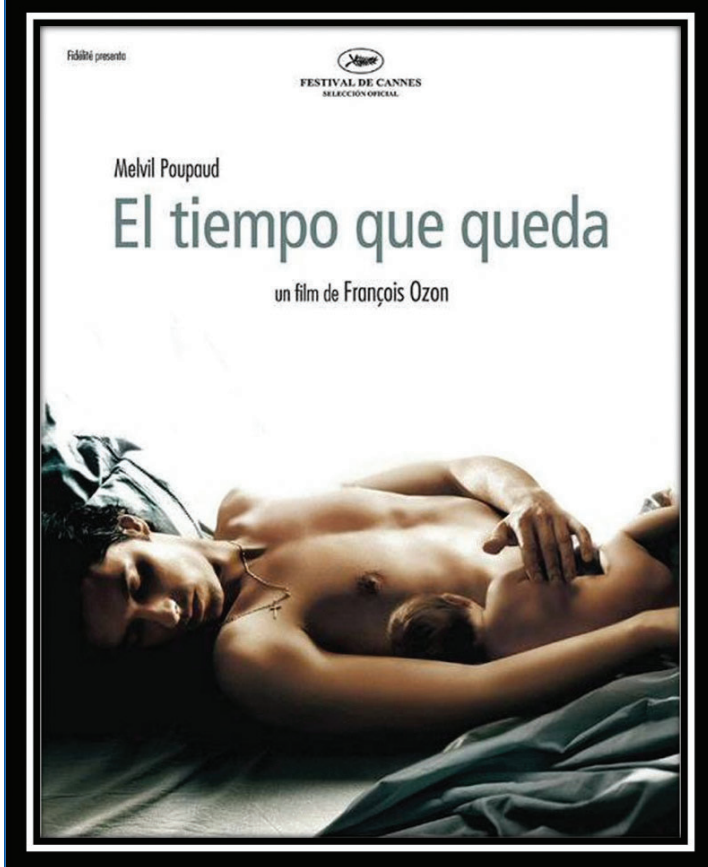

es de ira contra todo y todos los que le rodean, sin que el entorno conozca el motivo real de esta actitud. Cuando la soledad es más fuerte que su ira es cuando confiesa su enfermedad, primero a su abuela y luego a su novio.

\section{Gritos y susurros (Ingmar Bergman, 1972).}

\section{Suecia (Fig. 6)}

Cuenta los últimos días de Agnes (Harriet Andersson), una enferma de cáncer de útero en fase terminal. Ella es soltera y vive con una sirvienta, pero en esta fase de su vida también la cuidarán sus hermanas Karin (Ingrid Thulin) y María (Liv Ullman). El gran director sueco vuelve en esta obra (en este caso, con un excepcional cuarteto de mujeres) a temas recurrentes en su filmografía: la muerte, la fe y la incomunicación entre las personas.

\section{La vida (Jean-Pierre Améris, 2001). Francia (Fig. 7)}

Dimitri (Jacques Dutronc) Ilega a La Maison, un lugar destinado a enfermos donde la medicina tiene poco que hacer con enfermos como él, afecto de un cáncer de estómago metastatizado. Allí conoce 


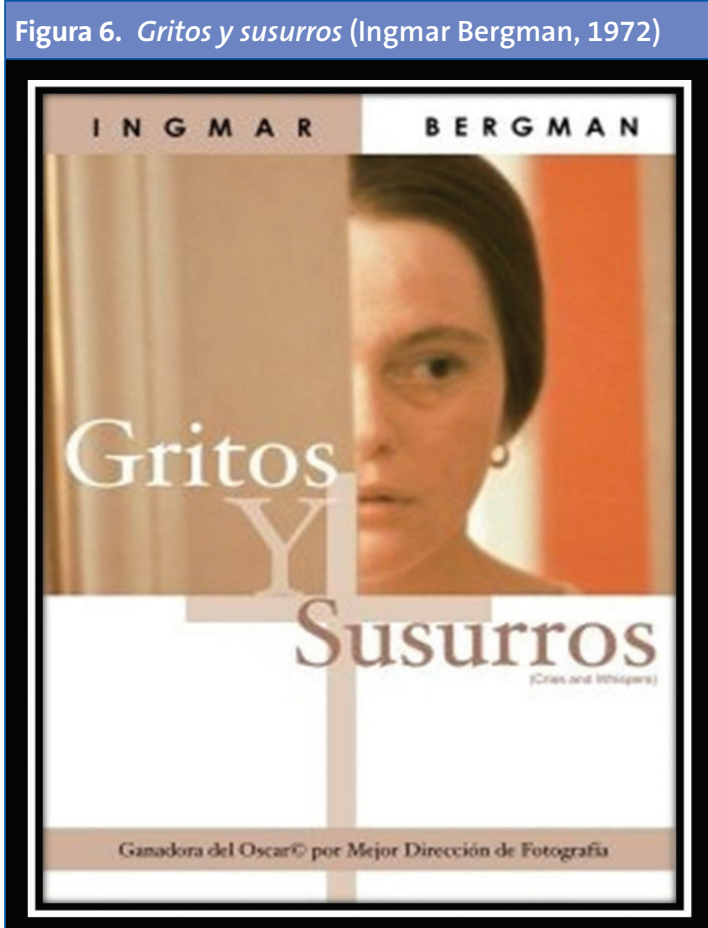

a Suzanne (Sandrine Bonnaire), una voluntaria que se dedica al acompañamiento de enfermos. El encuentro de ambos va a provocar el cambio de ella y la aceptación de él; y vivirán en el tiempo que les resta una intensa historia de amor y de felicidad. Al final de ese tiempo se habrá cumplido que la muerte de Dimitri implica también la vida de Suzanne. Es toda una exaltación a la necesidad de saber vivir aunque se esté desahuciado.

Las invasiones bárbaras (Denys Arcand, 2003).

Canadá (Fig. 8)

Rémy (Rémy Girard), un profesor con cáncer de hígado terminal, se encuentra internado en un hospital en Montreal. Tiene dificultades para aceptar la realidad de su inminente muerte y encontrar un momento de paz antes del final, especialmente porque tiene razones para lamentarse de ciertos aspectos de su pasado. Su hijo - del que se había distanciado-, su ex mujer, sus ex amantes y sus viejos amigos irán a reunirse con él para compartir sus últimos momentos, en los que discutirán sobre filosofía, política, pasado sexual y explotación intelectual. Y el podrá decir, finalmente: "Ha sido un placer vivir esta modesta vida en vuestra compañía mis queridos amigos. Y me llevo vuestras sonrisas conmigo".

Figura 8. Las invasiones bárbaras (Denys Arcand, 2003)
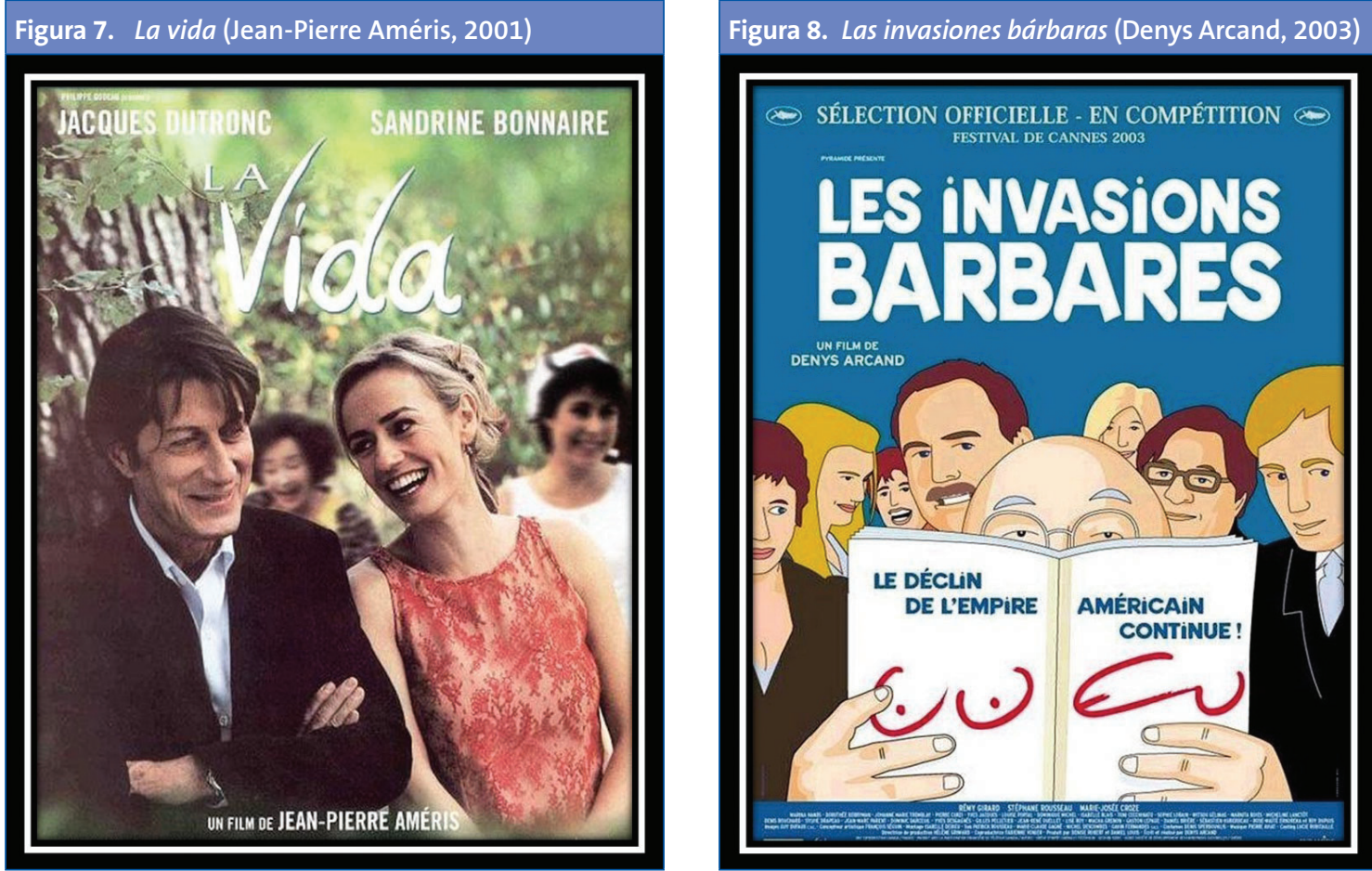


\section{Mi vida sin mi (Isabel Coixet, 2003). España (Fig. 9)}

Ann (Sarah Polley) es una joven casada y con dos hijas que vive una existencia anodina, pero su vida cambia cuando recibe la noticia de que tiene un cáncer de ovario con metástasis. Es en ese momento de final de camino, dado el mal pronóstico de su enfermedad, cuando su vida deja de ser gris y recupera un impulso vital en el que se propone completar una lista de cosas que hacer antes de morir.

Quimio (Pawel Lozinski, 2009). Polonia (Fig. 10)

Película documental en el hospital de día de una clínica oncológica de Varsovia, donde hombres y mujeres, ancianos, adultos y niños intercambian conversaciones, consejos y preocupaciones. Un documento donde lo ordinario se vuelve extraordinario, donde los primeros planos son los protagonistas: una sonrisa o una lágrima, una mirada de ilusión o de tristeza, una conversación esencial o fútil... y todo entre las paredes de un hospital y con enfermos oncológicos reales, y todo alrededor de cáncer y la vida. Menos de una hora de duración de pura emoción, emoción a la que nos tiene acos-

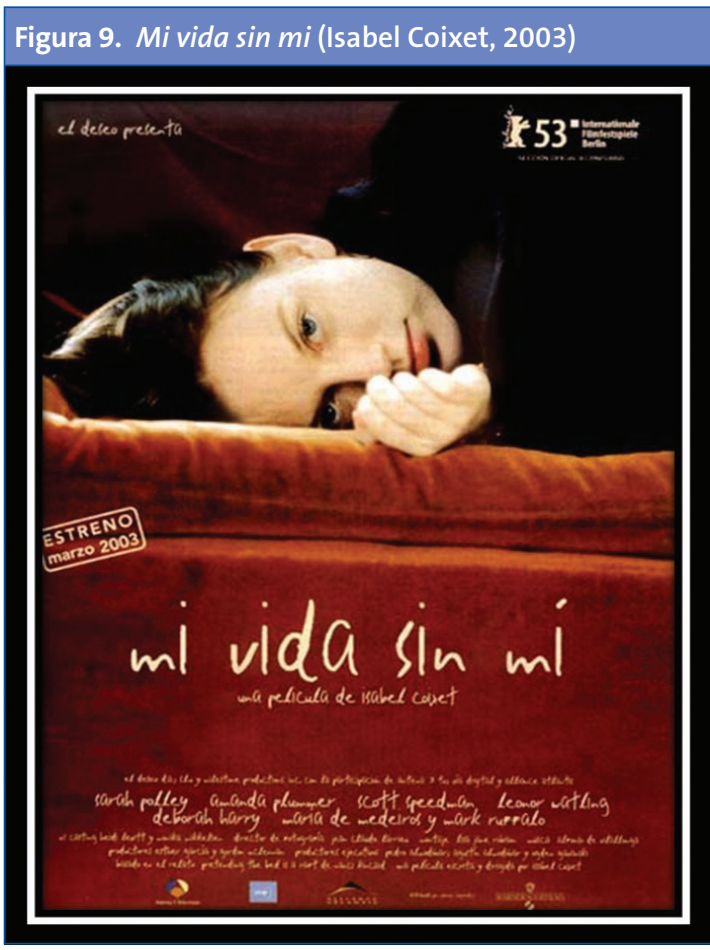

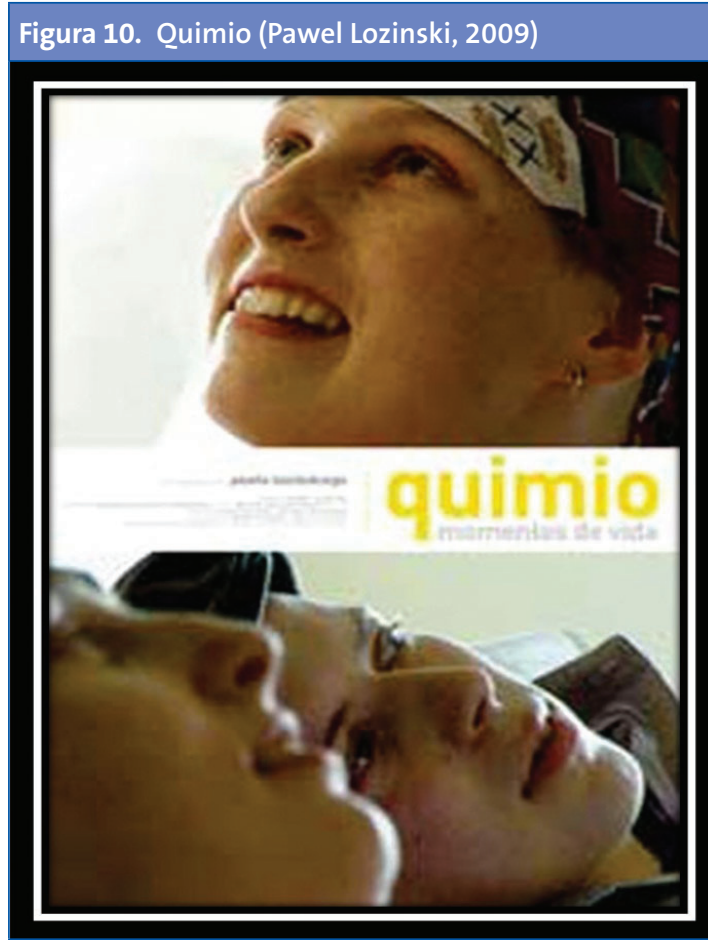

tumbrados los documentales de Pawel Lozinski: aquí nos permite compartir las experiencia reales de los pacientes con cáncer, sus dudas, ilusiones, esperanzas y miedos. Quimio se convierte en un testimonio sobre el cáncer y un reto: vencer la batalla.

\section{Stopped on Track (Andreas Dresen, 2011).} Alemania (Fig. 11)

Una radiografía de un cáncer terminal a través de un hiperrealismo casi documental en el que se nos narra la historia de Frank (Milan Peschel), un padre de familia de 44 años al que diagnostican un tumor cerebral inoperable. Los días están contados y, siendo consciente de la proximidad de la muerte, echa una mirada en profundidad a todo lo que va a dejar atrás: a su mujer Simone (Steffi Kühnert), a sus dos hijos, a sus amigos y a su amante. Frank nos narra a través de su iPhone el transcurrir de su enfermedad (y los crecientes síntomas) y las fases de su tratamiento (con la radioterapia y la quimioterapia como aliados) en un crudo drama que funciona como crónica vital de la enfermedad irreversible. Drama existencial y humano, lleno de 
Figura 11. Stopped on Track (Andreas Dresen, 2011)

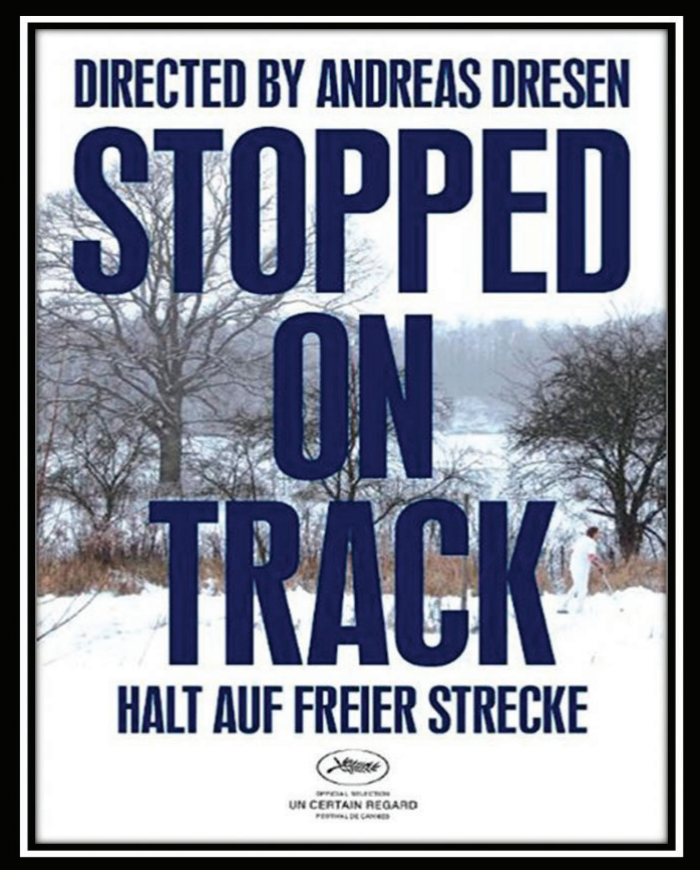

amargura, desesperanza y esperanza. Es la historia de una familia que se enfrenta a una gran pérdida y donde todos los planes se truncan: los proyectos de trabajo, la nueva casa, los viajes soñados, la música, etc. Pero la vida sigue, y deciden seguirla en el hogar, donde cada día es un pequeño adiós. Stopped on Track es una historia extrema, fuera de lo común y que para algunos es, paradójicamente, un canto a la vida.

\section{A MODO DE COMENTARIOS FINALES, A MODO DE COLOFÓN}

Confirmamos las conclusiones del estudio de IcartIsern et al. ${ }^{(19)}$ en el que se indica que, globalmente, el tipo de cáncer más tratado en el cine son las neoplasias hematológicas, principalmente leucemias. Entre el resto de entidades oncológicas, muy diversas, sí podemos apreciar una cierta mayor presencia de los tumores con mayor incidencia en el hombre (cáncer de pulmón) y en la mujer (cáncer de mama), con una presencia no desdeñable de tumores del sistema nervioso central, cáncer de próstata y cáncer de páncreas.
El cine también permanece fiel a la realidad en cuanto a la edad de presentación. En los pacientes adultos se concentran los cánceres ginecológicos (mama, ovario, útero, próstata, etc.) y en los niños y adolescentes, tal como veremos en nuestro próximo artículo ${ }^{28}$, predominan las leucemias, losn tumores cerebrales y los tumores óseos.

Es en aquellas películas sobre cáncer consideradas "argumentales" donde apreciamos una mayor y mejor descripción de la clínica asociada al tumor, así como de las intervenciones diagnóstico-terapéuticas y valoraciones pronósticas. Es cierto que la descripción de pruebas complementarias asociadas a los avances tecnológicos en estas enfermedades se aprecia más en las películas realizadas en el inicio del siglo XXI que en las anteriores.

Destacable es la imagen que estas películas transmiten de los profesionales sanitarios (médicos y enfermeras, principalmente), que varían en el grado de implicación científica con la enfermedad y emocional con el enfermo, así como en el manejo de la relación profesional-paciente. Es así que el cine se comporta, de forma destacable, como un magnífico espejo donde realizar un análisis interno (debilidades y fortalezas) y externo (amenazas y oportunidades) de nuestra profesión: en el entorno visual que nos proporciona la imagen se puede a prender a reforzar lo positivo y a modificar lo negativo. Digamos que, globalmente, el debate se establecerá entre lo "que no se debe hacer" en la atención de un paciente con cáncer y que debemos evitar (actitudes frías y distantes con el paciente, no considerar cada consulta como una consulta "sagrada", dar una información técnica y en un lugar inapropiado, etc.) y lo "que se debe hacer" y debemos potenciar (cuidar el lenguaje verbal y no verbal, trabajar la empatía y la implicación, analizar la atención al enfermo y sus familiares, etc.).

La mirada del cine al cáncer nos devuelve arte (no siempre, pues algunas películas son de escasa calidad cinematográfica, más en la línea del sentimentalismo fácil que del sentimiento profundo), ciencia (no siempre, pues en la mayoría de las películas consideradas "puntuales" y en alguna de las "relevantes" hay más espectáculo que rigor, donde 
la presencia del cáncer puede ser una mera anécdota en el guión) y, sobre todo, conciencia.

Conciencia y capacidad de pensamiento y reflexión, es una constante en casi todas las películas de esta temática. Porque es habitual de estas películas colocar a sus estrellas al borde de la vida, con una enfermedad terminal y preparadas para afrontar un fin inevitable: Bette Davis en Amarga victoria, Ali MacGraw en Love Story, William Hurt en El doctor, Diane Keaton en La habitación de Marvin, Jeremy Irons en La caja china, Susan Sarandon en Quédate a mi lado, Meryl Streep en Cosas que importan, Wynona Ryder en Otoño en Nueva York, Emma Thompson en Amar la vida, Jacques Dutronc en La vida, Kevin Kline en La casa de mi vida, Sarah Polley en Mi vida sin mí, Jürgen Vogel en La

\section{BIBLIOGRAFÍA}

1. Fresnadillo Martínez MJ, Diego Amado C, García Sánchez E, García Sánchez JE. Metodología docente para la utilización del cine en la enseñanza de la microbiología médica y las enfermedades infecciosa. Rev Med Cine. 2005;1:17-23.

2. Shapiro J. Literature and the arts in medical education. Fam Med. 2000;32:157-8.

3. Blasco PG. Literature and movies for medical students. Fam Med. 2001;33:426-8.

4. Whitman N. A poet confronts his own mortality: what a poet can teach medical students and teacher. Fam Med. 2000;32:673-4.

5. Blasco PG, Moreto G, Levites MR. Teaching Humanities through Opea: leading medical students to reflective attitudes. Fam Med. 2005;37:18-20.

6. Ber R, Alroy $G$. Twenty years of experience using trigger films as teaching tools. Acad Med. 2001;76:656-8.

7. Rabinowitz D, Melzer-Geva M, Ber R. Teaching the cultural dimensions of the patient-physician relationship: a novel approach using didactic trigger films. Med Teach. 2002;24:181-5.

8. SelfDJ, Baldwin DC. Teaching medical humanities through film discussions. J Med Humanit. 1990;11:23-9.

9. Self DJ, Baldwin DC, Olivarez M. Teaching medical ethics to first-year students by using film discussion to develop their moral reasoning. Acad Med. 1993; 68:383-5. suerte de Emma, Morgan Freeman y Jack Nicholson en Ahora o nunca, Saffron Burrows en The Guitar, Adam Sanler en Hazme reír... y una de las peor paradas ha sido Debra Winger en dos ocasiones: $L a$ fuerza del cariño y Tierras de penumbra.

El cine, una oportunidad para hablar con arte, ciencia y conciencia de la enfermedad oncológica y, sobre todo, de los pacientes con cáncer y su entorno. Una oportunidad para mejorar la relación profesional-paciente, para mejorar la humanización y para abrir el debate bioético.

\section{CONFLICTO DE INTERESES}

Los autores declaran no presentar conflictos de intereses en relación con la preparación y publicación de este artículo.
10. Wilt DI, Evans GW, Muenchen R, Guegold G. Teaching with entertainment films: an empathetic focus. J Psycosoc Nurs Ment Health Serv. 1995;33:6-15.

11. García Sánchez JR, Fresnadillo MU, García Sánchez E. El cine en la docencia de las enfermedades infecciosas y la microbiología clínica. Enferm Infecc Microbiol Clin. 2002;20:403-6

12. González Blasco P, Roncoletta AFT, Moreto G, Levites MR, Janaudis MA. Medicina de familia y cine: un recurso humanístico para educar la afectividad. Aten Primaria. 2005;36:566-72.

13. González de Dios J. Cine y Pediatría: una oportunidad para la docencia y la humanización en nuestra práctica clínica. Madrid: Exlibris Ediciones S.L.; 2012.

14. Domingo Moratalla T. Bioética y cine. De la narración a la deliberación. Madrid: San Pablo y Universidad Pontificia de Comillas; 2011.

15. Moratal Ibáñez LM. El cine y su mirada holística y humanitaria de las enfermedades. Rev Med Cine. 2007;3:85-6.

16. Mirón Canelo JA, Iglesias De Sena H, Alonso Sardón M. Valoración de los estudiantes sobre su formación en la Facultad de Medicina. Educ Med. 2011;14:221-8.

17. Baños Díez JE. ¿Cuál debe ser el grosor del estudio? La enseñanza de las sutilezas de la relación médicopaciente mediante obras literarias y películas comerciales. Rev Med Cine. 2007;3:159-65.

18. González de Dios J. Cine y Pediatría (I): una oportunidad para la docencia y la humanización en nuestra 
práctica clínica. Rev Pediatr Aten Prim. 2010;12:299313.

19. González de Dios J. Cine y Pediatría (II): infancia y adolescencia en las pantallas de cine de todo el mundo. Rev Pediatr Aten Prim. 2010;12:e21-e46.

20. Cappelletti GL, Sabelli MJG, Tenutto MA. ¿Se puede enseñar mejor? Acerca de la relación entre el cine y la enseñanza. Rev Med Cine. 2007;3:87-91.

21. Icart-Isern MT, Rosas-García MR, Sanfeliu-Cortes V, Viñas-Llebot H, Fernández-Ortega MP, Icart MC. El cáncer en el cine. Un recurso para los profesionales de salud. Educ Med. 2009;12:239-46.

22. García Sánchez JE, Trujillano Martín I, García Sánchez E. Medicina y cine ¿Por qué? Rev Med Cine. 2005;1:1-2.

23. Loscos J, Baños JE, Loscos F, de la Cámara J. Medicina, Cine y Literatura: una experiencia docente en la Universitat Autónoma de Barcelona. Rev Med Cine. 2006;2:138-42.

24. Baños Díez JE, Aramburu Beltrán JF, Sentí Clapés M. Biocinema: la experiencia de emplear películas co- merciales con estudiantes de Biología. Rev Med Cine. 2005;1:42-6.

25. Muñoz-Crego A, Santos Rodríguez Y, Seoane Prado R. MicroDeCine: la docencia de la Microbiología Clínica en la Universidad de Santiago de Compostela mediante el cine. Rev Med Cine. 2009;5:87-91.

26. González Blasco P, Pinheiro TRSP, Ulloa-Rodríguez MF, Angulo-Calderón NM. El cine en la formación ética del médico: un recurso pedagógico que facilita el aprendizaje. Pers Bioet. 2009;13:114-27.

27. Blasco PG, Moreto G, Roncoletta AFT, Levites MR, Janaudis MA. Using movie clips to foster learners' reflections.: improving education in the affective domain. Fam Med. 2006;38:94-6.

28. González de Dios J, Tasso Cereceda M, Ogando Díaz B. La mirada del cine al cáncer (II): cuando la Oncología pediátrica es la protagonista. Rev Pediatr Aten Primaria. 2012;14:xx-xx. 